\title{
Curcumin phytosomal softgel formulation: Development, optimization and physicochemical characterization
}

\author{
AHMED N. ALLAM ${ }^{1, *}$ \\ IBRAHIM A. KOMEIL ${ }^{2}$ \\ OSSAMA Y. ABDALLAH ${ }^{1}$ \\ ${ }^{1}$ Faculty of Pharmacy \\ University of Alexandria, Egypt \\ ${ }^{2}$ Medizen Pharmaceutical Industries \\ Alexandria, Egypt
}

Accepted June 25, 2015

\begin{abstract}
Curcumin, a naturally occurring lipophilic molecule can exert multiple and diverse bioactivities. However, its limited aqueous solubility and extensive presystemic metabolism restrict its bioavailability. Curcumin phytosomes were prepared by a simple solvent evaporation method where free flowing powder was obtained in addition to a newly developed semisolid formulation to increase curcumin content in softgels. Phytosomal powder was characterized in terms of drug content and zeta potential. Thirteen different softgel formulations were developed using oils such as Miglyol 812, castor oil and oleic acid, a hydrophilic vehicle such as PEG 400 and bioactive surfactants such as Cremophor EL and KLS P 124. Selected formulations were characterized in terms of curcumin in vitro dissolution. TEM analysis revealed good stability and a spherical, self-closed structure of curcumin phytosomes in complex formulations. Stability studies of chosen formulations prepared using the hydrophilic vehicle revealed a stable curcumin dissolution pattern. In contrast, a dramatic decrease in curcumin dissolution was observed in case of phytosomes formulated in oily vehicles.
\end{abstract}

Keywords: curcumin, phospholipid, phytosomes, bioavailability, lipids, softgels

\section{INTRODUCTION}

Curcumin (CUR) found in the turmeric rhizomes can exert multiple and diverse bioactivities, including antioxidant, antitumor, anti-inflammatory, antidiabetic, antirheumatic, wound healing, antiviral, hepatoprotective, and anti-HIV activity (1). Despite the potential biological effects of CUR, poor bioavailability in both rodents and humans, as reported in several pharmacokinetic studies (2), demands high doses to reach therapeutic drug levels. Detectable serum levels were not achieved until doses of up to $3.6 \mathrm{~g}$ were used; consequently, a $3.6 \mathrm{~g}$ daily dose of oral CUR was used in the phase II clinical trial in patients with advanced colorectal cancer (3).

\footnotetext{
*Correspondence; e-mail: allam@alexu.edu.eg
} 
Keeping in mind the promise of CUR as a therapeutically active agent and its poor oral absorption due to its limited aqueous solubility and extensive presystemic metabolism, it is necessary to develop a new formulation of CUR that could augment its oral absorption and enhance its therapeutic activity.

To improve the bioavailability of CUR, numerous approaches and technologies have been investigated over the past decades, including the use of solid dispersion (4), copolymeric micelles (5), polymeric nanoparticles (6), lipid based nanoparticles (7), self microemulsion (8), liposomes (9) and complexation with phospholipids (10, 11). All these approaches are expected to provide bioavailability enhancement and improved absorption in the intestinal tract.

Phytosomes are considered to be a safe and convenient delivery system, able to enhance oral bioavailability of poorly water soluble drugs. Their safety is related to the similarity between their structural components and the lipid content of mammalian cell membranes (12-14). Phytosomes show a better stability profile than liposomes due to the unique chemical bond interaction between phospholipid molecules and polyphenolic compounds (15).

Meriva $^{\mathrm{TM}}$ of Indena Company is the only patented commercial product containing CUR formulated as a complex with phospholipids (16). It is available as a hard gelatin capsule containing $100 \mathrm{mg}$ of CUR.

Softgel technology could be utilized to improve oral absorption of CUR phytosomes, along with increasing the available dose. The softgel dosage form has several advantages over other oral dosage forms, such as delivering a liquid matrix intended to solubilize and enhance oral bioavailability of a poorly soluble compound. In addition, it can protect the encapsulated compound against oxygen and light, and it is able to readily disperse in gastric juices. From the industrial point of view, this technology is beneficial since it minimizes potential generation of dust during manufacturing and thereby improves the safety of production personnel $(17,18)$. Softgels have received enormous consumer acceptance due to the ease of swallowing and unpleasant taste masking (19). Use of formulations containing natural and/or synthetic lipids in addition to excipients with reported bioactive effect on metabolism could provide a good opportunity to manage the poor gastrointestinal absorption associated with CUR.

The main goal of this study was to prepare a CUR phospholipid complex in dry powder using a simple method, and then formulate it in a final dosage form utilizing the advantages of softgel technology. Moreover, development of a new semisolid fill formulation was aimed to increase drug loading in order to improve patient compliance and adherence. The formulated CUR phytosomes were characterized in terms of in vitro dissolution behavior and selected formulations were further assessed for different physicochemical properties before and after storage.

\section{EXPERIMENTAL}

\section{Materials}

Curcumin powder was purchased from Shenzhen Chemrider, China. The soy phosphatidylcholine (SPC) phospholipid, Lipoid S 100, was kindly supplied by Lipoid Co., Ger- 
many. Kollisolv P 124 (KLS P124), Polyoxyl 35 castor oil (CRM EL) and Polyoxyl 40 hydrogenated castor (CRM RH40) were purchased from BASF, USA. Soya bean oil and capric/ caprilic triglyceride (Miglyol 812) were purchased from Gattefossé Corp., USA. Polyethylene glycol 400 (PEG 400) was a sample gift from Galaxy, India. Colloidal silicon dioxide (Aerosil 200) and sodium lauryl sulphate were purchased from Evonik Degussa, UK. All other reagents and chemicals were of analytical grade.

\section{Preparation of the curcumin-phospholipid complex (CUR-SPC complex)}

In the preparation of the curcumin-phospholipid complex, the method reported by Maiti et al. (10) and Gupta el al. (11) was utilized with slight modification. Briefly, CUR and SPC S 100 in a molar ratio of 1:1 were dissolved in $20 \mathrm{~mL}$ of dichloromethane in a round bottom flask. This mixture was refluxed for $2 \mathrm{~h}$ at room temperature, stirred by a magnetic stirrer (IKA T25, Germany) and poured into a beaker containing colloidal silicon dioxide powder (Aerosil 200) in the same amount as SPC. The solvent was evaporated by placing the beaker in a controlled temperature water bath and using a mixer (IKA T25, Germany) at $40-45^{\circ} \mathrm{C}$ for 4 hours.

\section{Analysis of CUR content in the prepared CUR-SPC complex}

Sample of $100 \mathrm{mg}$ of test powder was sonicated in $100 \mathrm{~mL}$ of methanol in a volumetric flask for $15 \mathrm{~min}$. After filtration of the test solution, $5 \mathrm{~mL}$ of the filtrate was diluted with 100 $\mathrm{mL}$ of the same solvent. Samples were analyzed spectrophotometrically (Shimadzu, Japan) at a wavelength of $420 \mathrm{~nm}$.

\section{Zeta potential measurement}

Zeta potential (ZP) of the prepared CUR-SPC complex was measured using the dynamic light scattering (DLS) technique (Malvern Zetasizer, Malvern Instruments, UK). Samples were dispersed in distilled water and sonicated for $15 \mathrm{~min}$. After sonication, samples were diluted with distilled water (1:10) before measurements.

\section{Curcumin solubility study}

An excess quantity of CUR was added to different oils and surfactants in a tightly capped conical flask and was constantly agitated at $100 \mathrm{rpm}$ and $37^{\circ} \mathrm{C}$ for $24 \mathrm{~h}$ in a reciprocating water bath (Bunsen, India). Samples were centrifuged (4000 rpm, $15 \mathrm{~min}$ ) after $24 \mathrm{~h}$ equilibrium and aliquots of supernatant were diluted to appropriate concentrations with acetone. The samples were analyzed using a spectrophotometer (Shimadzu, Japan) at a wavelength of $420 \mathrm{~nm}$ with acetone as a blank.

\section{Preparation of softgel fill formulations of CUR-SPC complex}

According to the lipid formulation classification system, class IIIA was selected to formulate the CUR-SPC complex as softgel using a hydrophilic surfactant (i.e., HLB > 12) and triglyceride oil. Butylated hydroxy toluene was added as antioxidant to guard against autoxidation of polyoxyethylene moiety for CRM EL or KLX P 124. 


\section{Preparation of CUR-SPC complex powder as a fill formulation}

Dispersion method (20) was applied to prepare lipid fill formulations where hydrophilic surfactant was mixed with triglyceride oil for 5 min using a magnetic stirrer (IKA T25, Germany). Prior to encapsulation, CUR phytosomal powder was geometrically mixed with a hydrophilic surfactant/triglyceride blend in a ratio of 1:2 up to formation of a homogenous mass. Compositions of lipid fill formulations are shown in Table I.

Table I. Composition of CUR-SPC complex powder softgel fill formulations

\begin{tabular}{lccccccccc}
\hline Material (mg) & F1 & F2 & F3 & F4 & F5 & F6 & F7 & F8 & F9 \\
\hline CUR-SPC complex & 500 & 500 & 500 & 500 & 500 & 500 & 500 & 500 & 500 \\
BHT & & 0.2 & 0.2 & 0.2 & 0.2 & 0.2 & 0.2 & 0.2 & 0.2 \\
CRM EL & - & 100 & 200 & 400 & 200 & - & - & - & - \\
CRM RH 40 & - & - & - & - & - & 200 & 200 & - & - \\
KLS P 124 & - & - & - & - & - & - & - & 200 & 400 \\
Soybean oil & - & - & - & - & 799.8 & - & 799.8 & - & - \\
Miglyol 812 & 1000 & 889.8 & 799.8 & 599.8 & - & 799.8 & - & 799.8 & 599.8 \\
\hline
\end{tabular}

\section{Preparation of CUR-SPC complex as a semisolid fill formulation}

CUR and SPC S 100 in a molar ratio of 1:1 were dissolved and refluxed in $60 \mathrm{~mL}$ of dichloromethane for 2 hours. The prepared solution was placed in a controlled temperature water bath adjusted to $45^{\circ} \mathrm{C}$ for $4-5$ hours up to formation of a semisolid mass. A hydrophilic or lipophilic vehicle was added to the prepared semisolid complex in a 1:1.5 ratio and mixed mechanically at room temperature until formation of a liquefied mass. Composition of the lipophilic/hydrophilic vehicles of the CUR-SPC complex semisolid fill formulations is shown in Table II.

Table II. Composition of lipophilic/hydrophilic vehicles of CUR-SPC complex semisolid fill formulations

\begin{tabular}{lcccc}
\hline Material $(\mathrm{mg})$ & F10 & F11 & F12 & F13 \\
\hline CUR & 200 & 200 & 200 & 200 \\
SPC S100 & 400 & 400 & 400 & 400 \\
BHT & 0.2 & 0.2 & 0.2 & 0.2 \\
KLS P- 124 & 360 & 360 & 360 & - \\
CRM EL & - & - & - & 360 \\
Miglyol 812 & 539.8 & - & - & - \\
Oleic acid & - & 539.8 & - & - \\
Castor oil & - & - & 539.8 & - \\
PEG 400 & - & - & - & 539.8 \\
\hline
\end{tabular}




\section{In vitro dissolution studies}

In vitro dissolution test was carried out according to USP 32 (21) for CUR capsule dissolution, where $900 \mathrm{~mL}$ of $1 \%$ sodium lauryl sulphate (SLS) aqueous solution was used as dissolution medium with the application of apparatus II (Hanson, USA) at $100 \mathrm{rpm} .40 \mathrm{mg}$ of curcumin, curcumin phytosomal powder and different lipid/semisolid fill formulations (in terms of CUR) were filled in a hard gelatin capsule and attached to a sinker prior to the start of the dissolution test. After $60 \mathrm{~min}$, a $10 \mathrm{~mL}$ aliquot was withdrawn, filtered, diluted and analyzed spectrophotometrically at a wavelength of $420 \mathrm{~nm}$.

In vitro dissolution studies of selected formulations were performed in order to obtain the dissolution profile. Aliquots were withdrawn at time intervals of 30, 60, 120 and 180 min. Samples were diluted and analyzed spectrophotometrically at $420 \mathrm{~nm}$.

\section{Transmission electron microscopy (TEM)}

Morphological examination of phytovesicles of the prepared CUR phytosomal powder and some selected CUR-SPC complex formulations was carried out using TEM (model JEM-100S microscope, Jeol, Japan). Samples were diluted with a $0.01 \%$ SLS aqueous solution (1:20) and sonicated for $10 \mathrm{~min}$. A drop of the resultant phospholipid complex dispersions was placed onto a carbon-coated copper grid, leaving a thin liquid film. The film was air-dried and then imaged (22).

\section{Stability studies}

Selected formulations, F9, F10 and F13, were stored in air filled soft gelatin capsules for 3 months at $25{ }^{\circ} \mathrm{C}$ and $65 \%$ relative humidity. Samples were monitored for any change in the physical state during the storage period. Formulations were assessed for their in vitro dissolution profile, phytovesicular morphology and ZP before and after storage.

\section{RESULTS AND DISCUSSION}

\section{Preparation of CUR phytosomes}

The CUR-SPC complex was prepared by the method reported by Maiti et al. (10) and Gupta et al. (11) with some modifications. Briefly, CUR and SPC S 100 were refluxed in a common organic solvent able to solubilize both ingredients for a definite time, followed by removal of the organic solvent by a rotary evaporator. Industrially, the mentioned method could not be applied to produce a commercial form of the CUR-SPC complex powder to be used as a dosage form. To produce curcumin phytosomal powder, a very low density powder (Aerosil 200) was selected among different fillers as the most appropriate filler. Solvent was evaporated completely after $4-5$ hours at $45-50{ }^{\circ} \mathrm{C}(24)$. The complex flakes formed were mechanically ground to produce a fine and flowable powder.

\section{Analysis of CUR content}

Analysis was carried out for total curcuminoids in test samples. Results revealed that the amount of total curcuminoids in the prepared phytosomal powder was $19.06 \%$. The 
content of total curcuminoids in the CUR-SPC complex powder depends on the amount of filler used in the preparation; 2 parts of Aerosil 200 were used to make the ratio of CUR, SPC and filler 1:2:2. The amount of curcuminoids in the CUR-SPC complex powder may be increased by decreasing the amount of filler, which requires further examinations.

\section{Measurement of zeta potential (ZP)}

The magnitude of zeta potential indicates the degree of electrostatic repulsion between similarly charged particles, which influences the stability of colloidal dispersions. Phytosomes carry a negative charge due to the presence of the SPC phosphate group in water environment at neutral $\mathrm{pH}$ (25). Results revealed that $\mathrm{ZP}$ of the prepared CUR phytosomes was $-35.4 \pm 2.45 \mathrm{mV}$, which indicates good stability. It has been suggested that high absolute $\mathrm{ZP}$ values $( \pm 30 \mathrm{mV}$ ) may serve as a partial indicator of physical stability to ensure creation of a high energy barrier against rupture or coalescence of the formed globules (26).

\section{Curcumin solubility study}

Saturated solubility of CUR in water could not be detected because of spectrum shift from the wavelength of $420 \mathrm{~nm}$ to $280 \mathrm{~nm}$ due to its poor stability and decomposition under neutral-basic conditions (23). CUR solubility studies were carried out in different aqueous and non-aqueous media and the results are shown in Fig. 1.

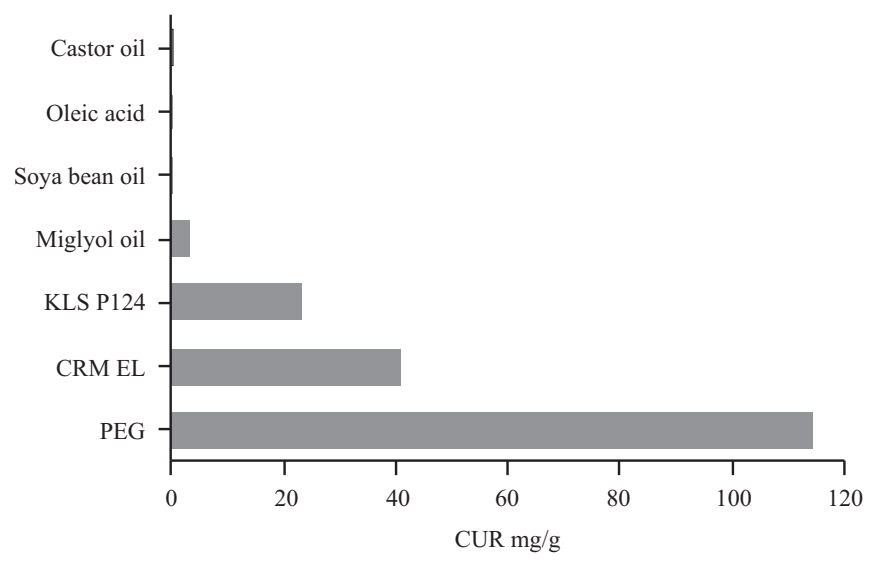

Fig. 1. Solubility of CUR in different vehicles and surfactants.

CUR solubility in Miglyol 812, soya bean oil and castor oil was 3.56, 2.64 and $0.267 \mathrm{mg}$ $\mathrm{g}^{-1}$, respectively. Hydrophilic surfactants (CRM EL and KLS P 124) caused a significant increase in saturated solubility of CUR, reaching 41 and $23 \mathrm{mg} \mathrm{g}^{-1}$, respectively. On the other hand, CUR solubility in PEG and castor oil of 114.6 (7) and $0.267 \mathrm{mg} \mathrm{g}^{-1}$ (8), resp., was reported in the literature. 


\section{Preparation of softgel fill formulations of the CUR-SPC complex}

There are many advantages of selecting softgels as a dosage form for the development of curcumin phytosomal powder, one of them being the ability to increase the dose of CUR in softgels to $200 \mathrm{mg}$ CUR per capsule compared to only $100 \mathrm{mg}$ CUR per marketed hard gelatin capsule. Another advantage is the use of several bioactive excipients, like poloxamers, PEG, CRM RH 40 and CRM EL, because of their reported inhibitory effect on CYP3A4 $(27,28)$ affecting the intestinal metabolism of CUR $(29)$ and hence promoting its systematic bioavailability.

Type IIIA lipid formulation containing hydrophilic surfactant and medium chain triglyceride oil was selected to formulate the CUR-SPC complex powder in softgels.

\section{Preparation of CUR-SPC complex powder as a softgel fill formulation}

Different hydrophilic surfactants were used at different concentrations to optimize the fill formulation in order to enhance the CUR dissolution profile pursuant to pharmacopoeial requirements. The optimum ratio between the CUR-SPC complex powder and oil to be encapsulated in softgel was found to be 1:2, as seen in F1. CRM EL was used in F2, F3, and F4 at concentrations of 10, 20 and $40 \%(\mathrm{~m} / \mathrm{m})$, respectively, in the presence of Miglyol 812 as oily vehicle to study the effect of different CRM EL concentrations on CUR dissolution. An increase in the fill formulation viscosity was observed with the increase of the CRM EL concentration. On the other hand, in the formulation F5, $20 \%(\mathrm{~m} / \mathrm{m})$ CRM EL was used in the presence of soya bean oil instead of Miglyol 812 to show the effect of a different type of oily vehicle on the dissolution rate of CUR-SPC complex powder.

In formulations F6 and F7, CRM RH $40(20 \%(\mathrm{~m} / \mathrm{m}))$ was utilized instead of CRM EL to study the effect of using another surfactant from the same chemical group with higher HLB value on the dissolution of CUR formulated with different oily vehicles, Miglyol 812 and soya bean oil, respectively.

In formulations F8 and F9, KLS P 124 was used in concentrations of (20 and $40 \%, \mathrm{~m} / \mathrm{m})$, respectively, with Miglyol 812 to study the effect of different concentrations of liquid poloxamer on the dissolution profile of CUR from the CUR-SPC complex.

\section{Preparation of CUR-SPC complex semisolid fill formulations}

Utilizing the semisolid form of the CUR-SPC complex is considered to be a new method for the preparation of CUR-SPC complex softgels. This method provides several advantages over the conventional method, including the use of a liquid vehicle instead of fillers to fluidize the semisolid form of the prepared complex, doubling the CUR content in the softgel up to $200 \mathrm{mg}$, few preparation steps and minimal equipment, along with a small chance of material loss during the preparation of the softgel fill formulation. To preserve the CUR-SPC complex structure in a fluidized form, the used liquefied vehicle must not affect complex integrity, which could be achieved by using either a lipophilic or hydrophilic vehicle with hydrophilic surfactant. Components of CUR-SPC complex semisolid fill formulations are shown in Table II. 


\section{In vitro dissolution studies}

Dissolution profile of the CUR phytosomal powder failed to achieve pharmacopoeial tolerance and formulation development was required to enhance the CUR dissolution profile to comply with the pharmacopoeial limits (i.e., not less than $75 \%$ after 1 hour in 1 $\%$ SLS aqueous solution).

Results of in vitro dissolution studies of softgel formulations of CUR-SPC complex powder (F1-F9) and CUR-SPC complex semisolid fill formulation (F10-F13) are graphically shown in Fig. 2. Formulation F1 showed relatively low CUR dissolution, reaching only $13 \%$ after $60 \mathrm{~min}$, which could be ascribed to the high affinity of CUR-SPC complex powder towards lipophilic vehicle (pure miglyol oil).

Dissolution was enhanced by increasing the concentration of hydrophilic surfactant (34, 38 and $80 \%$ of CUR dissolved) where 10,20 and $40 \%(\mathrm{~m} / \mathrm{m})$ CRM EL was used in the preparation of F2, F3 and F4, respectively. Although formulation F4 gave better dissolution results than F2 and F3, it was excluded from further studies due to its high viscosity.

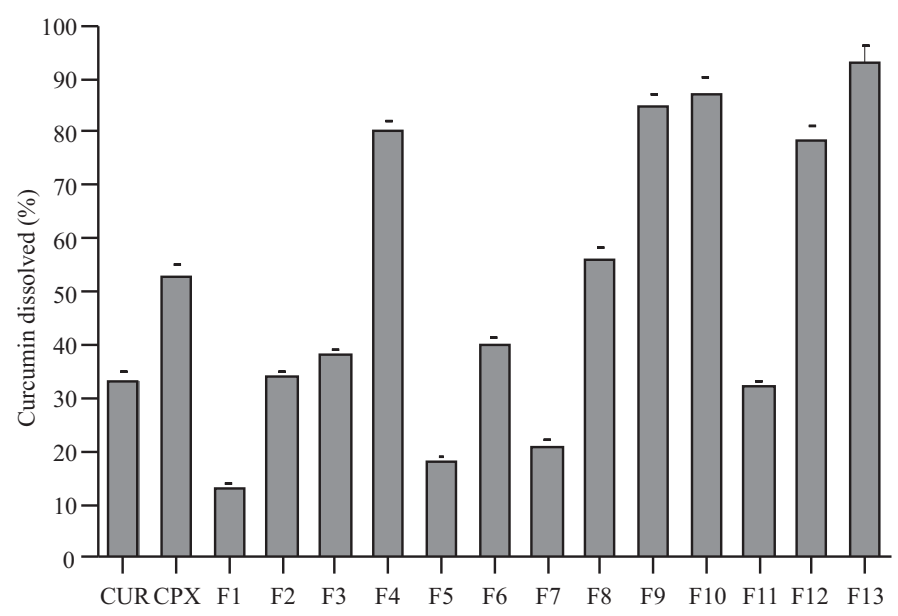

Fig. 2. In vitro dissolution of CUR powder, CUR-SPC complex (CPX) and different CUR fill formulations after $60 \mathrm{~min}$ in $1 \%$ SLS aqueous solution.

To study the effect of different surfactants on the dissolution of the CUR-SPC complex, CRM EL, CRM RH 40 and KLS P 124 were used in formulations F3, F6 and F8, respectively. It was observed that there was no difference between $20 \%(\mathrm{~m} / \mathrm{m}) \mathrm{CRM} E L$ and $20 \%$ $(\mathrm{m} / \mathrm{m})$ CRM RH 40 while using $20 \%(\mathrm{~m} / \mathrm{m})$ KLS P 124 showed better results $(56 \%$ of CUR dissolved after $60 \mathrm{~min}$ ). Therefore, higher concentration of KLS P 124 was examined in formulation F9 which gave the highest CUR dissolution, reaching $85 \%$ after $60 \mathrm{~min}$, which was acceptable according to USP pharmacopoeial tolerance.

To select the oily vehicle, soybean oil and miglyol oil were examined in formulations with the same type of surfactant and at the same concentrations as shown for using $20 \%$ CRM EL in F3 and F5 and $20 \%$ CRM RH 40 in F6 and F7, respectively. Dissolution of the 
CUR-SPC complex was greatly enhanced when using Miglyol 812 rather than soybean oil; this may be due to better solubility of CUR in Miglyol 812 than in soybean oil, as shown in Fig. 1.

For CUR-SPC complex semisolid fill formulations, lipophilic and hydrophilic vehicles were examined as carriers for the prepared complex. Results for lipophilic vehicles showed that using miglyol oil in formulation F10 rendered better dissolution behavior than formulations F11 and F12 containing oleic acid and castor oil, respectively. This may be due to the relatively high CUR solubility in Miglyol $812\left(3.56 \mathrm{mg} \mathrm{g}^{-1}\right)$ compared to oleic acid and castor oil. On the other hand, using PEG 400 and CRM EL as hydrophilic vehicles resulted in the highest dissolution among lipophilic formulations, which could be also attributed to high CUR solubility in PEG (114.6 $\left.\mathrm{mg} \mathrm{g}^{-1}\right)$. Based on the above mentioned results, F9, F10 and F13 were selected for further in vitro dissolution and stability studies. Fig. 3 shows CUR dissolution enhancement by preparing its complex with SPC; dissolution of the latter was further improved in formulations F9, F10 and F13. Encapsulation of the CUR-SPC complex semisolid formulation in softgels gave better dissolution than CUR-SPC complex powder, which could be attributed to the liquefied form of the complex used in our new method of preparation.

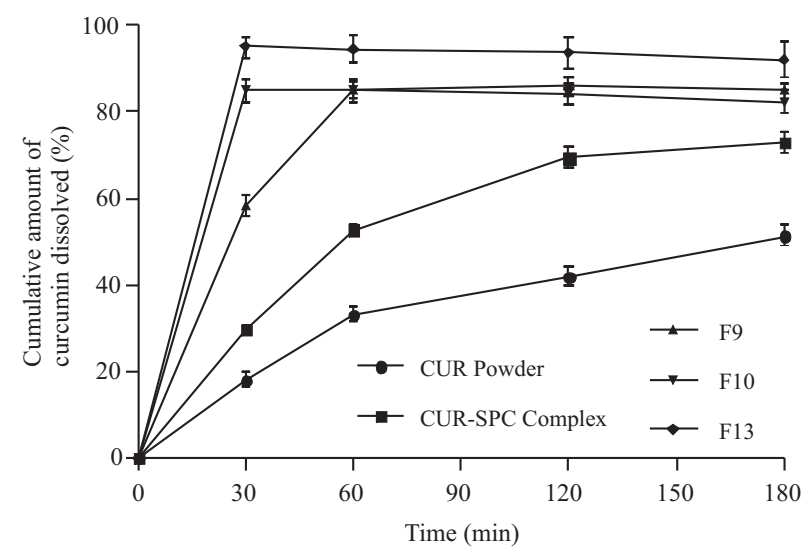

Fig. 3. Comparative dissolution profile of pure CUR, CUR-SPC complex, formulations F9, F10 and F13 in $1 \%$ SLS aqueous solution.

\section{Morphological examination of CUR phytosomes}

TEM analysis revealed good stability and spherical, self-closed structure of CUR phytosomes in complex formulations (Fig. 4a and 4b). Complex formulations did not change the amphiphilic properties and water dispersion associated with phospholipids (12). Saraf et al. (30) suggested that upon interaction with water, the hydrophilic heads of phospholipids are oriented in the direction of the water compartment while the lipophilic tails are oriented away from the water, which is in agreement with our findings. TEM micrographs of formulation F13 in Fig. 4c show opaque vesicles with a regular surface, which may be 
referred to the CUR-SPC complex solubilized in PEG 400. Continuous dynamic movement of solubilized CUR in PEG 400 supported its ability to appear inside the hollow phospholipid structure and gave denser vesicles compared to the prepared phytosome powder (Fig. 4a).
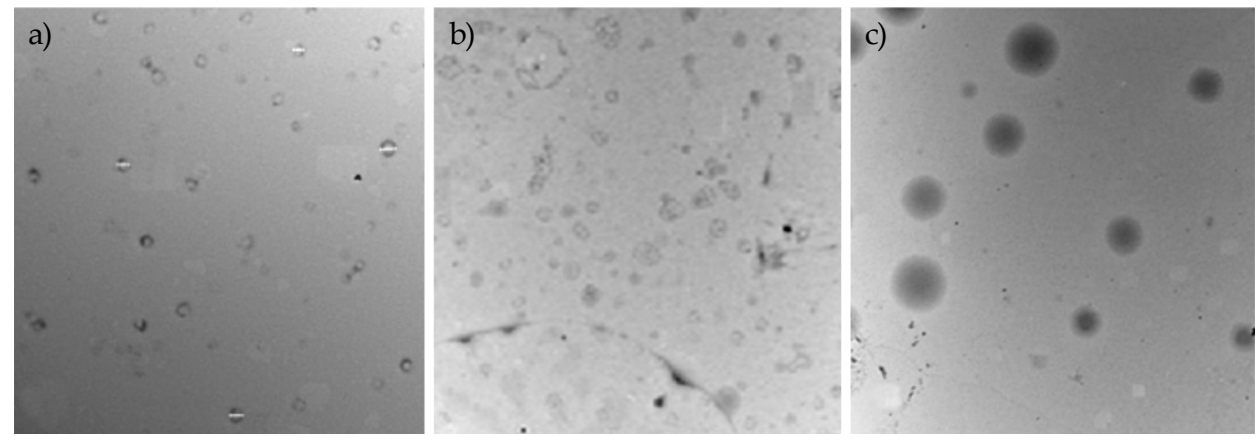

Fig. 4. Transmission electron micrographs of CUR-SPC complex (a), formulations F10 (b) and F13 (c) (magnification 10,000 X).

\section{Stability studies of CUR-SPC fill formulations}

As shown in Figs. 5a and b, the dissolution rate of the CUR-SPC complex was sharply decreased after 3 months of storage for formulations F9 and F10 compared to time zero, which could be ascribed to limited solubility of CUR in oily vehicles used in the preparation of such formulations as well as to affinity of SPC towards the oily vehicle, leading to the salting out of CUR. Salting out of CUR may affect the integrity of the CUR-SPC complex, leading to a decrease in CUR dissolution.

In contrast, the dissolution profile of formulation F13, shown in Fig. 5c, did not change after 3 months of storage. This may be explained by the compatibility of PEG 400 and the CUR-SPC complex. Thus, the application of PEG 400 as hydrophilic vehicle to formulate the CUR-SPC complex did not affect the CUR-SPC complex integrity.

TEM micrographs of formulation F10 support our suggestions about the CUR salting out from the CUR-SPC complex. Fig. 6a shows transparent vesicles with thin irregular membranes (phospholipid layers) and a small black spot in the middle of each vesicle (salted out CUR). These findings support the idea of possible destruction of the CUR-SPC complex, which in turn affects the dissolution behavior of formulation F 10. In contrast, no change in phytosome morphology was observed before and after 3 months of storage of formulation F13 (Figs. 4c and 6b, respectively), which is in agreement with the dissolution results.

The integrity of the phytosome in formulation F13 was also confirmed by zeta potential measurements. No change in ZP was observed for aqueous dispersion of F13 (-38.8 \pm $3.48 \mathrm{mV}$ ) compared to that for the CUR-SPC complex $(-35.4 \pm 2.45 \mathrm{mV})$, which indicates good stability. It is therefore concluded that PEG 400 does not affect the CUR-SPC complex 

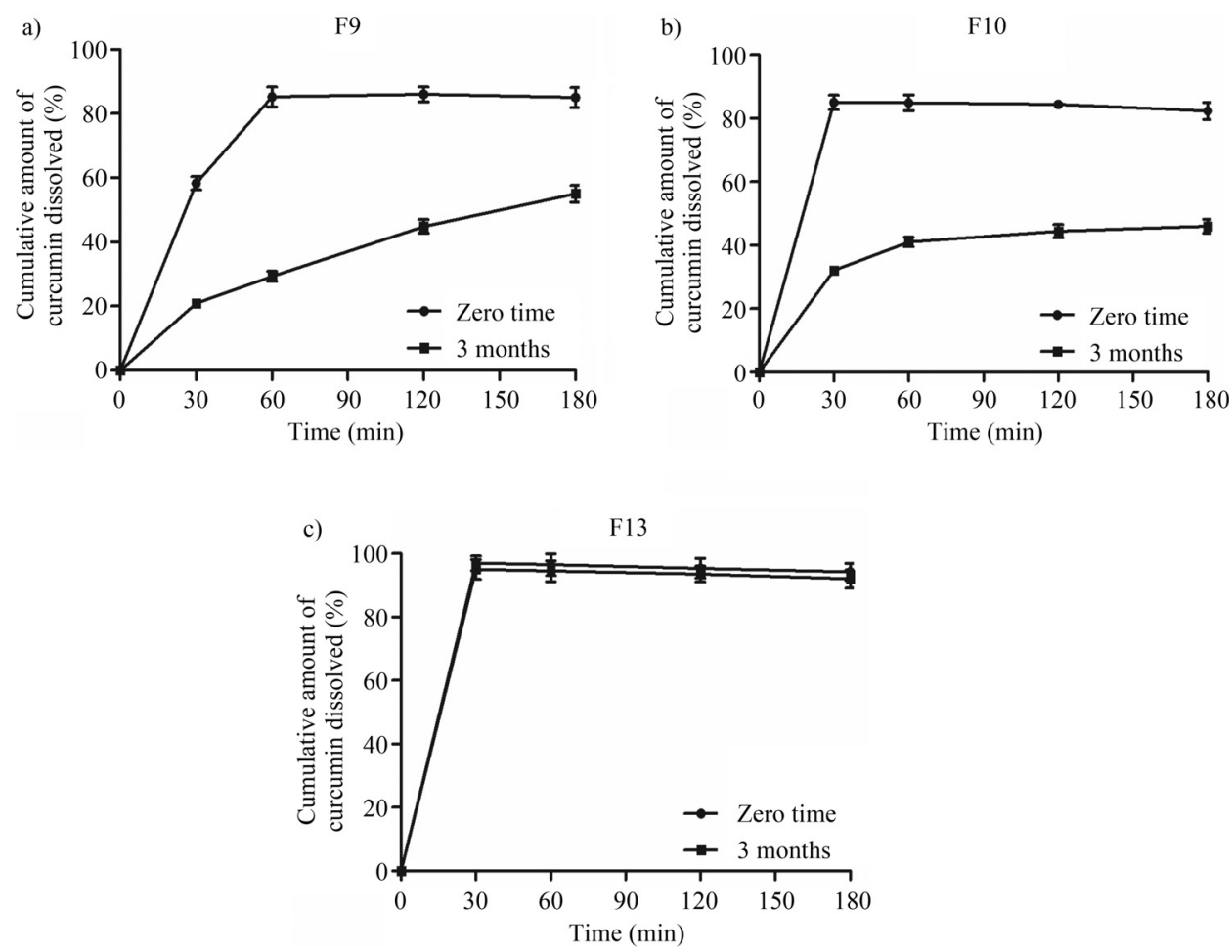

Fig. 5. Comparative dissolution profile of curcumin from formulatios F9, F10 and F13 at time zero and after 3 months of storage.
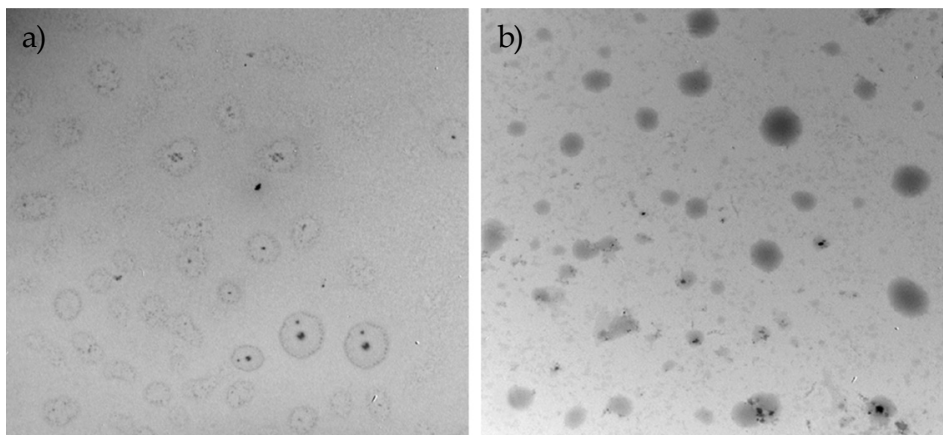

Fig. 6. Transmission electron micrographs of formulations F10 (a) and F13 (a) after 3 months of storage.

integrity, which is in agreement with the findings obtained by both dissolution studies and TEM analysis. 


\section{CONCLUSIONS}

We have successfully developed a CUR-SPC complex using a simple, low cost, industrially applicable method. Preliminary studies showed that softgel can be a promising dosage form for hydrophobic and extensively metabolized compounds in GIT like curcumin, enabling their oral application and patient compliance.

Utilizing soft gelatin capsules as an oral dosage form provides advantages over hard gelatin capsules through their ability to increase drug loading in the formulation and its bioavailability by using different bioactive excipients. When formulating phytosomes in a soft gelatin capsule, attention must be paid to the selection of excipients (i.e., oils or surfactants) to avoid salting out phenomena due to the difference in solubility of active herbal drugs in different excipients.

\section{REFERENCES}

1. R. K. Maheshwari, A. K. Singh, J. Gaddipati and R. C. Srimal, Multiple biological activities of curcumin: a short review, Life Sci. 78 (2006) 2081-2087; DOI: 10.1016/j.lfs.2005.12.007.

2. R. Sharma, C. Ireson, R. Verschoyle, K. Hill, M. Williams, C. Leuratti, M. Manson, L. Marnett and W. Steward, Effects of dietary curcumin on glutathione-S-transferase and malondialdehyde-DNA adducts in rat liver and colon mucosa: relationship with drug levels, Clin. Cancer Res. 7 (2001) 1452-1458.

3. R. A. Sharma, S. A. Euden, S. L. Platton, D. N. Cooke, A. Shafayat, H. R. Hewitt, T. H. Marczylo, B. Morgan and D. Hemingway, Phase I clinical trial of oral curcumin: Biomarkers of systemic activity and compliance, Clin. Cancer Res. 10 (2004) 6847-6854.

4. A. Paradkar, A. A. Ambike, B. K. Jadhav and K. R. Mahadik, Characterization of curcumin-PVP solid dispersion obtained by spray drying, Int. J. Pharm. 271 (2004) 281-286; DOI: 10.1016/j. ijpharm.2003.11.014.

5. M. H. Leung, H. Colangelo and T. W. Kee, Encapsulation of curcumin in cationic micelles suppresses alkaline hydrolysis, Langmuir. 24 (2008) 5672-5675; DOI: 10.1021/la800780w.

6. T. H. Kim, H. H. Jiang, Y. S. Youn, C. W. Park, K. K. Tak, S. Lee, H. Kim and S. Y. Jon, Preparation and characterization of water-soluble albumin bound curcumin nanoparticles with improved antitumor activity, Int. J. Pharm. 403 (2011) 285-591; DOI: 10.1016/j.ijpharm.2010.10.041.

7. Y. B. Pawar, H. Purohit, G. Valicherla, B. Munjal, S. V. Lale, S. Patel and A. K. Bansal, Novel lipid based oral formulation of curcumin: development and optimization by design of experiments approach, Int. J. Pharm. 436 (2012) 617-623; DOI: 10.1016/j.ijpharm.2012.07.031.

8. J. Cui, B. Yu, Y. Zhao, W. Zhu, H. Li and H. Lou, Enhancement of oral absorption of curcumin by self-microemulsifying drug delivery systems, Int. J. Pharm. 371 (2009) 148-155; DOI: 10.1016/j. ijpharm.2008.12.009.

9. C. G. Chen, T. D. Johnston, H. Jeon, R. Gedaly, P. P. McHugh, T. G. Burke and D. Ranjan, An in vitro study of liposomal curcumin: stability, toxicity and biological activity in human lymphocytes and Epstein-Barr virus-transformed human B cells, Int. J. Pharm. 366 (2009) 133-139; DOI: 10.1016/j.ijpharm.2008.09.009.

10. K. Maiti, K. Mukherjee, A. Gantait, B. Saha and P. K. Mukherjee, Curcumin-phospholipid complex: Preparation, therapeutic evaluation and pharmacokinetic study in rats, Int. J. Pharm. 330 (2007) 155-163; DOI: 10.1016/j.ijpharm.

11. N. K. Gupta and V. K. Dixit, Bioavailability enhancement of curcumin by complexation with phosphatidylcholine, J. Pharm. Sci. 100 (2011) 1987-1995; DOI: 10.1002/jps.22393. 
12. J. Zhang, Q. Tang, X. Xu and N. Li, Development and evaluation of a novel phytosome-loaded chitosan microsphere system for curcumin delivery, Int. J. Pharm. 448 (2013) 168-174; DOI: 10.1016/j. ijpharm.2013.03.021.

13. P. Shivanand and P. Kinjal, Phytosomes: Technical revolution in phytomedicine, Int. J. PharmaTechRes. 2 (2010) 627-631.

14. J. Patel, R. Patel, K. Khambholja and N. Patel, An overview of phytosomes as an advanced herbal drug delivery system, Asian J. Pharm. Sci. 4 (2009) 363-371.

15. A. Semalty, M. Semalty, B. S. Rawat, D. Singh and M. S. Rawat, Pharmacosomes: the lipid-based new drug delivery system, Expert Opin. Drug Deliv. 6 (2009) 599-612; DOI: 10.1517/17425240902967607.

16. http://www.phytosomes.info/public/meriva.asp

17. H. I. Benza and W. L. Munyendo, A review of progress and challenges in soft gelatin capsules formulations for oral administration, Int. J. Pharm. Sci. Rev. Res. 10 (2011) 20-24.

18. R. P. Gullapalli, Soft gelatin capsules (softgels), J.Pharm. Sci. 99 (2010) 4109-4121; DOI: 10.1002/ jps.22151.

19. W. J. Jones, Softgels: consumer perceptions and market impact relative to other oral dosage forms, Adv. Ther. 17 (2000) 213-221.

20. L. L. Schramm, Emulsions, Foams, and Suspensions: Fundamentals and Applications, Wiley, Weinheim 2005.

21. M. Brewster, R. Vandecruys, J. Peeters, P. Neeskens and G. Verreck, Comparative interaction of 2-hydroxypropyl cyclodextrin and sulfobutylether-cyclodextrin with itraconazole: phase-solubility behavior and stabilization of supersaturated drug solutions, Eur. J. Pharm. Sci. 34 (2008) 94-103; DOI: 10.1016/j.ejps.2008.02.007.

22. M. S. Freag, Y. S. Elnaggar and O. Y. Abdallah, Lyophilized phytosomal nanocarriers as platforms for enhanced diosmin delivery: optimization and ex vivo permeation, Int. J. Nanomed. 8 (2013) 2385-2397; DOI: 10.2147/IJN.S45231.

23. F. Zhang, G. Y. Koh, D. P. Jeansonne, J. Hollingsworth, P. S. Russo, G. Vicente, R. W. Stout and Z. Liu, A novel solubility-enhanced curcumin formulation showing stability and maintenance of anticancer activity, J. Pharm. Sci. 100 (2011) 2778-89; DOI: 10.1002/jps.22512.

24. Soybean Phospholipids, in Recent Trends for Enhancing the Diversity and Quality of Soybean (Ed. D. Krezhova), InTech 2011, pp. 483-450.

25. Z. Hou, Y. Li, Y. Huang, C. Zhou, J. Lin, Y. Wang, F. Cui, S. Zhou, M. Jia, S. Ye and Q. Zhang, Phytosomes loaded with mitomycin C-soybean phosphatidylcholine complex developed for drug delivery, Mol. Pharm. 10 (2013) 90-101; DOI: 10.1021/mp300489p.

26. M. A. Elsheikh, Y. S. Elnaggar, E. Y. Gohar and O. Y. Abdallah, Nanoemulsion liquid preconcentrates for raloxifene hydrochloride: optimization and in vivo appraisal, Int. J. Nanomed. 7 (2012) 3787-3802; DOI: 10.2147/IJN.S33186.

27. X. Ren, X. Mao, L. Si, L. Cao and H. Xiong, Pharmaceutical excipients inhibit cytochrome P450 activity in cell free systems and after systemic administration, Eur. J. Pharm. Biopharm. 70 (2008) 279-288; DOI: 10.1016/j.ejpb.2008.03.019.

28. X. Ren, X. Mao, L. Si, L. Cao and J. Qiu, Nonionic surfactants are strong inhibitors of cytochrome P450 3A biotransformation activity in vitro and in vivo, Eur. J. Pharm. Biopharm. 36 (2009) 401-411; DOI: 10.1016/j.ejps.2008.11.002.

29. B. Wahlang, Y. B. Pawar and A. K. Bansal, Identification of permeability-related hurdles in oral delivery of curcumin using the Caco-2 cell model, Eur. J. Pharm. Biopharm. 77 (2011) 275-282; DOI: 10.1016/j.ejpb.2010.12.006.

30. S. A. Saraf, Applications of novel drug delivery system for herbal formulations, Fitoterapia $\mathbf{8 1}$ (2010) 680-689; DOI: 10.1016/j.fitote.2010.05.001. 